\title{
La filière tomate de transformation à Haouaria en Tunisie : prédominance de la forme industrielle déterritorialisée
}

\author{
Lamia $\operatorname{Arfa}^{1,2, *}$ et Mohamed Elloumi ${ }^{2}$ \\ ${ }^{1}$ Université de Carthage, Institut national agronomique de Tunis, 1082 Tunis, Tunisie \\ ${ }^{2}$ Université de Carthage, Institut national de recherche agronomique de Tunisie, LR16INRAT07, Laboratoire d'économie rurale, rue Hédi \\ Karray, 1004 El Menzah, Tunisie
}

\begin{abstract}
Résumé - En Tunisie, la filière tomate de transformation joue un rôle majeur dans le développement socioéconomique local de certains territoires. La plaine de Haouaria, région historiquement spécialisée dans cette production industrielle, connaît depuis les années 2010 un processus de déterritorialisation se traduisant en amont par une plus grande vulnérabilité des agriculteurs. L'objectif de cet article est de présenter les variables explicatives de ce processus. Pour ce faire, nous avons mobilisé le cadre conceptuel de l'approche filière. Notre démarche méthodologique s'est appuyée sur une recherche bibliographique et documentaire, des enquêtes de terrain auprès d'agriculteurs et de consommateurs, des entretiens avec les acteurs institutionnels et les industriels et enfin des focus groupes avec différentes parties prenantes de la filière. L'article analyse la structure et les mécanismes de régulation de la filière et estime le poids du territoire dans son développement. Nos résultats montrent que la structure de cette filière est dominée par la forme industrielle, avec des entreprises dont la production est peu diversifiée et est constituée principalement de double concentré de tomate, produit de base du modèle de consommation alimentaire tunisien. Cette structure se caractérise par une forte asymétrie de pouvoir ainsi qu'une répartition inégale de la valeur entre les acteurs économiques de la filière. Des mécanismes de régulations ont été développés par les acteurs économiques et l'État afin de s'adapter à diverses contraintes internes en amont, telles que la baisse de disponibilité en eau et la saturation du marché. Cette reconfiguration se traduit par une forte dépendance de cette filière à d'autres régions pour son développement. Finalement, il apparaît que la déterritorialisation de cette filière résulte des régulations internes et externes, alors que les actions de territorialisation (diversification des produits, reconnaissance par des dispositifs de labellisation, etc.) restent timides et ont du mal à se structurer.
\end{abstract}

Mots clés : filière; tomate; territoire; territorialisation; Tunisie

\begin{abstract}
The tomato supply chain in Haouaria: predominance of the deterritorialized industrial form. In Tunisia, the tomato supply chain plays a major role in the local socio-economic development of certain territories. The plain of Haouaria, a region historically specialized in the tomato processing, has known since the 2010 s a process of deterritorialization resulting in a greater vulnerability of farmers. The objective of this article is to present the explanatory variables of this process. In order to do that, we mobilized the conceptual framework of the supply chain approach. Our methodology was based on documentary research, field surveys with farmers and consumers, interviews with institutional stakeholders and manufacturers and finally focus groups with various stakeholders in the sector. The article analyzes the structure, the regulatory mechanisms of the sector, and estimates the weight of the territory in its development. Our results show that the structure of this sector is dominated by the industrial form, with a production consisting mainly in tomato paste, the basic product of the Tunisian food consumption model. This structure is characterized by a strong asymmetry of power as well as an unequal distribution of value between stakeholders. Regulatory mechanisms have been developed by stakeholders and the government in order to adapt to various constraints such as decrease in water availability and market saturation. This reconfiguration results in a strong dependence of this supply chain on other regions for its development. Finally, it appears that the deterritorialization of the tomato-processing sector in the
\end{abstract}

\footnotetext{
*Auteur de correspondance : lamia.arfa@gmail.com
} 
study region is mainly the result of internal and external regulation methods, while territorialization actions such as product diversification or recognition through labeling mechanisms, remain weak and not yet structured.

Keywords: supply chain, tomato, territory, territorialization, Tunisia

\section{Introduction}

En 2020, pour la troisième année consécutive, la Tunisie a gardé le dixième rang mondial pour la production de tomates de transformation, avec une augmentation de $18 \%$ par rapport à l'année 2019. Cette production a ainsi atteint de 1,2 millions de tonnes. Elle a occupé 10000 producteurs pour une superficie totale de 16500 ha (GICA, 2020).

Les quantités de tomates transformées par les industries ( 1 million de tonnes) ont représenté $86 \%$ des quantités produites, le reste étant destiné au marché du frais et à la transformation artisanale ou familiale. $91 \%$ des quantités transformées par l'industrie, soit 943000 tonnes, ont produit 143000 tonnes de double concentré de tomate (DCT). Le DCT est obtenu par des procédés industriels d'extraction et de concentration de la pulpe de tomate à des taux de 28 à $30 \%$ de matière sèche soluble naturelle. Le DCT est le produit le plus important dans la consommation tunisienne de tomate et dérivés. En effet, avec une consommation annuelle moyenne de $10 \mathrm{~kg}$ de DCT par an (soit l'équivalent de $65 \mathrm{~kg}$ de tomates fraîches), les Tunisiens sont les premiers consommateurs mondiaux de DCT. La consommation annuelle moyenne totale est estimée à 100000 tonnes.

Par ailleurs, l'exportation du DCT est une activité régulière et régulatrice pour cette filière. Ainsi, durant la période 20082018, les exportations annuelles moyennes ont été de l'ordre de 13000 tonnes de DCT, soit $11 \%$ de la production totale. Ces exportations ont toutefois baissé à 3209 tonnes en 2019 (GICA, 2020) à cause de l'instabilité politique en Libye, principal client de la filière.

En Tunisie, la production des conserves de tomate représente $60 \%$ du secteur des conserves alimentaires et $90 \%$ de celui des conserves de fruits et légumes. En 2019, 24 usines se sont partagé l'activité de transformation de la tomate, dont 14 situées dans la région du Cap-Bon (GICA, 2020).

Le Cap-Bon a toujours été la plus importante région pour cette activité. La première usine de transformation de Tunisie y a été installée en 1903. Au sein de cette région, le gouvernorat de Nabeul, avec $32 \%$ des superficies cultivées en 2020, est le plus important gouvernorat pour cette culture.

Toutefois, depuis les années 2010, le manque d'eau et le développement de la production dans d'autres régions ont entraîné une baisse relative de l'importance de la culture de la tomate industrielle au sein de ce gouvernorat. En effet, en 2000-2005, le gouvernorat de Nabeul occupait $61 \%$ des superficies tunisiennes de tomates de transformation, alors que cette part n'était plus que de $31 \%$ en 2010-2015.

Face à la réduction de la part des superficies, nous assistons à une déterritorialisation de la filière avec la spécialisation de la région du Cap Bon dans la fourniture de plants pour les autres régions et le recours à d'autres bassins de production pour l'approvisionnement des usines de transformation.
Dans cet article, nous étudions les mutations à l'œuvre et les dynamiques de cette filière, en nous référant à un bassin de production particulier, celui de la plaine de Haouaria (Fig. 1).

Notre principal objectif est de préciser les caractéristiques de cette filière dans ce bassin.

Plus particulièrement, nous cherchons à répondre aux questions suivantes :

- Comment cette filière a-t-elle pu développer son potentiel de transformation et d'exportation, et ce malgré la forte baisse des superficies cultivées en tomate?

- Quels sont les mécanismes de régulation de cette filière et quels sont les rapports de force entre ses acteurs?

- Quel serait l'avenir probable de la filière dans le territoire de Haouaria?

\section{Matériels et méthode}

\subsection{Approche filière et territorialisation}

Pour répondre à ces questions, nous mobilisons l'approche filière telle que définie par Bencharif et Rastoin (2007). Cette approche filière est devenue, à partir des années 1960, un outil privilégié d'aide à la décision publique (Griffon, 2002). Elle a permis une meilleure compréhension des différents types de relations qui animent les agents économiques autour d'un produit (Champion, 2014). L'approche filière prend ainsi en compte tous les acteurs qui sont liés par un produit particulier. Plusieurs disciplines telles que l'économie industrielle, l'anthropologie, la sociologie et la géographie ont contribué au développement de l'analyse filière par des enrichissements successifs de ses outils d'analyse. Elle s'est également inspirée de l'approche systémique (Bencharif et Rastoin, 2007) qui considère la filière en tant que système caractérisé par une structure et un fonctionnement. La structure est composée par les acteurs de la filière qui entretiennent des relations en évolution. Le fonctionnement est décrit par des processus techniques, organisationnels (Temple et al., 2011) et de régulation. Il est sujet à des facteurs de changement-évolution et d'innovation de type technologique, social ou organisationnel (Madelrieux et al., 2017). L'analyse systémique en tant qu'outil d'étude des filières distingue les systèmes de production, d'échange et d'encadrement. Ces systèmes développent des relations techniques (intrant-produit), organisationnelles (degré d'intégration) et d'échange (type de concurrence).

Nous retenons également la notion de territorialisation telle que définie par Madelrieux et al. (2017). Le concept de filière, appliqué à l'étude d'un produit lié à un territoire, amène à observer et à analyser les relations méso-économiques, verticales et horizontales, entre agents. Il s'agit de prendre en considération non seulement les déterminants technico-économiques mais 


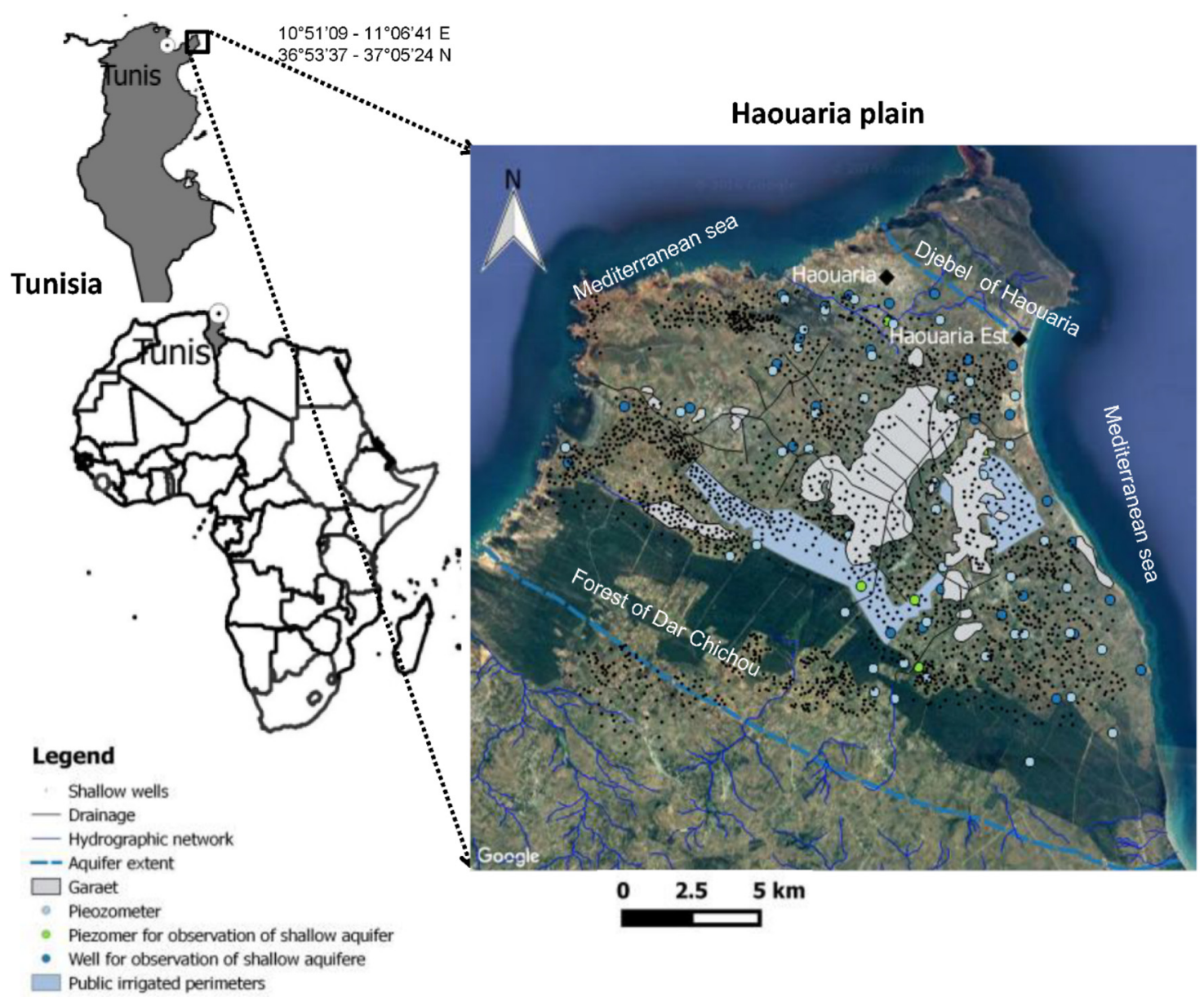

Fig. 1. Localisation de la plaine de Haouaria (source: Ferchichi et al., 2020).

Fig. 1. Location of the Haouaria plain (source: Ferchichi et al., 2020).

également spatio-temporels dans les décisions économiques des différents agents qui forment la filière considérée (Rastoin et Ghersi, 2010). La répartition géographique des différentes activités caractérise les dimensions locale, régionale ou nationale de la filière. Elle montre l'importance du territoire pour la filière. Les filières utilisent certaines ressources de leurs territoires, y opèrent une partie plus ou moins importante de leurs activités et y exercent des impacts socio-économiques et environnementaux.

Dans divers pays et régions, la gestion des filières a été caractérisée par la concentration des opérateurs de l'aval ainsi que l'éloignement des centres de décision des centres de production (Rastoin et Ghersi, 2010). En même temps, la connexion entre les opérateurs des filières et ceux des territoires est devenue de plus en plus faible (Manouvrier, 2008). Ceci a conduit à des filières productivistes, des produits standardisés et une fragmentation régionale de ces filières (Tozanli et El Hadad-Gauthier, 2007). L'approche filière est l'un des outils les plus utilisés pour l'étude des relations de connexion/déconnexion entre les opérateurs d'un produit particulier (Cheriet, 2015).

Historiquement, dans plusieurs pays développés, les filières agricoles ont connu une importante restructuration à travers la déconnexion plus ou moins forte entre les opérateurs des filières et ceux des territoires (Madelrieux et al., 2017).
Toutefois, dans beaucoup de pays en développement, la connexion a été plus stable et plus importante. L'étude d'une filière, l'identification de son territoire d'implantation et l'importance des lieux de production et d'usage des ressources locales renvoient à la territorialisation qui, plus qu'un lien au territoire, distingue les enjeux des acteurs et la notion de gouvernance alimentaire qui émerge de plus en plus comme dimension caractéristique de la filière (Temple et al., 2011). Les filières peuvent avoir un rôle moteur dans le développement de leur territoire avec des impacts socio-économique et environnementaux importants.

\section{2 Étude de cas de la plaine de Haouaria}

Au sein du gouvernorat de Nabeul, la plaine de Haouaria $\left(300 \mathrm{~km}^{2}\right)$ est composée des délégations de Haouaria et de Hammam Ghezaz. Située au nord de la forêt de Dar Chichou (Fig. 1), c'est un grand bassin de production de cultures industrielles. En 2017, cette plaine a fourni $61 \%$ des cultures industrielles du gouvernorat de Nabeul. En 2018, elle a représenté environ $20 \%$ des superficies en tomate de transformation de ce même gouvernorat. Néanmoins, ce bassin de production a enregistré une forte baisse des superficies en tomate à partir du début des années 2010 

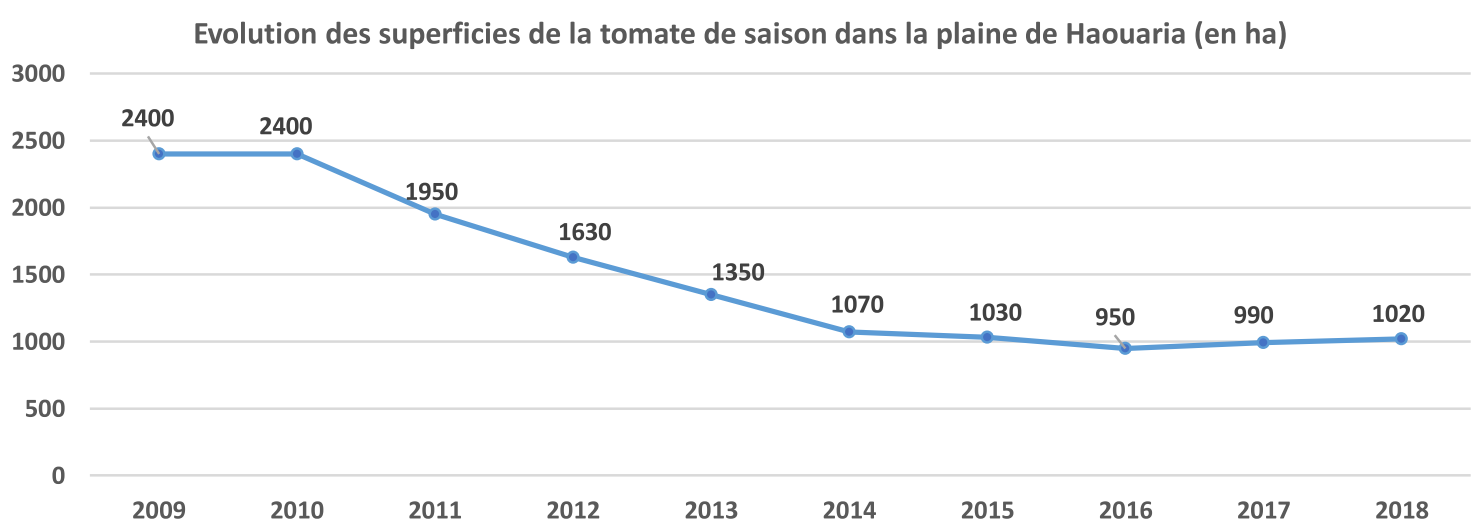

Fig. 2. Évolution de la superficie cultivée en tomate de transformation dans la plaine de Haouaria (source: CTV, 2019).

Fig. 2. Evolution of the cultivated area for processing tomatoes in the Haouaria plain (in hectares) (source: CTV, 2019).

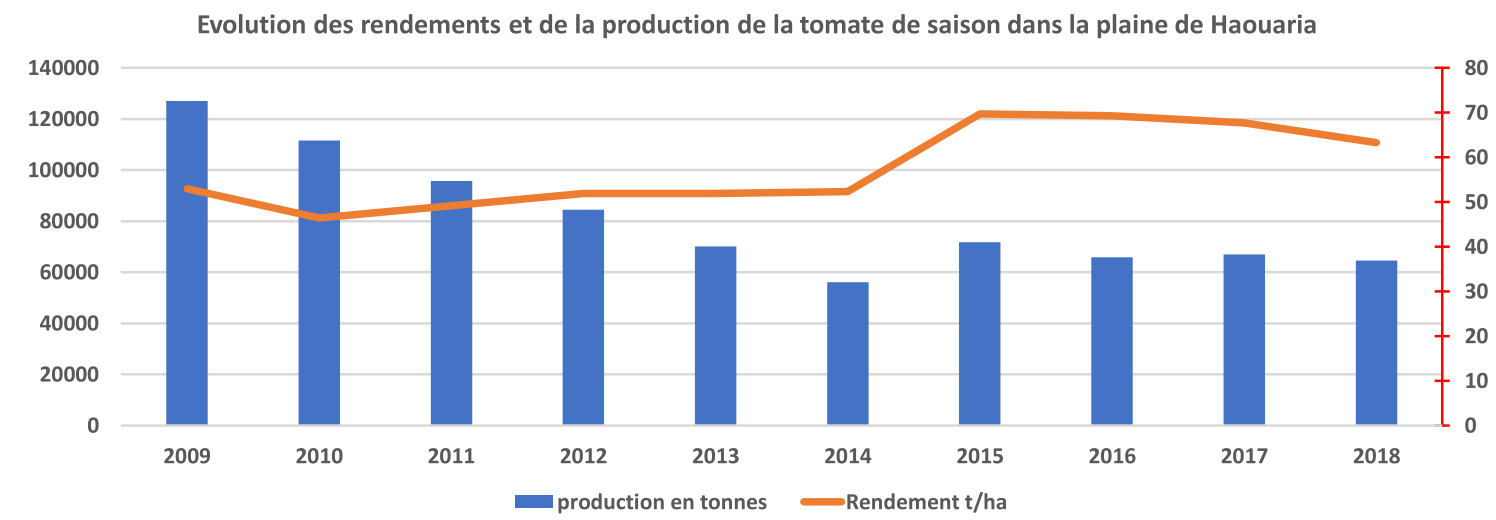

Fig. 3. Évolutions du rendement et de la production de la tomate de transformation dans la plaine de Haouaria (source: CTV, 2019).

Fig. 3. Evolution of yields and production of processing tomatoes in the Haouaria plain (source: CTV, 2019).

(Fig. 2). En effet, les cultures maraîchères et industrielles irriguées du bassin de Haouaria ont entraîné une forte consommation d'eau. En 2018, celle-ci a atteint $114 \%$ du potentiel renouvelable des nappes phréatiques et $120 \%$ de celui des nappes profondes de ce bassin (CGDR, 2019). La culture de la tomate a ainsi été impactée par la forte exploitation des ressources en eau et la baisse conséquente de leurs disponibilités (Ferchichi et al., 2020). L'État a alors instauré durant les campagnes 2010-2013 une subvention de $300 \mathrm{TND} / \mathrm{ha}$ ( 1 euro $=2,29$ dinars tunisiens fin 2013) pour encourager la baisse des superficies cultivées en tomates de transformation et la reconversion vers d'autres cultures moins consommatrices en eau. Par conséquent, les superficies en tomate pour la transformation ont baissé de moitié, dans la plaine, entre 2011 et 2018, pour se stabiliser à environ 1000 ha à partir de 2014 (Fig. 2). La forte baisse des superficies s'est traduite par une baisse conséquente de la production qui est passée de 119000 tonnes en 2009-2010 à 66000 tonnes en 2016-2018 (Fig. 3), soit 8,8\% de la production tunisienne. L'amélioration des rendements à partir de 2015 a toutefois compensé en partie la baisse des superficies (Fig. 3).

La tomate produite dans cette plaine est vendue et transformée sur place grâce à un tissu industriel formé par 4 usines de transformation dont la capacité, en 2018, était équivalente à $50 \%$ de celle du gouvernorat et à $25 \%$ de celle de la Tunisie (GICA, 2020)
L'activité industrielle y demeure donc importante avec une part élevée dans les exportations tunisiennes: $25 \%$ des exportations tunisiennes de DCT en 2018 (GICA, 2020). Avec une capacité de transformation installée qui dépasse la production locale, les usines ont de plus en plus recours à d'autres régions du pays pour leur approvisionnement. De plus, les fournisseurs de plants de tomates de la plaine approvisionnent aussi les autres régions. Cela témoigne d'un processus de déterritorialisation.

\subsection{Collecte de données}

Afin de pouvoir étudier la filière de la tomate de transformation de la plaine de Haouaria, nous avons mobilisé des données relatives aux structures et au fonctionnement des différents maillons de la filière. Il s'agit de données quantitatives sur les superficies, les rendements et les prix des tomates, sur les quantités produites, commercialisées et exportées de DCT et enfin sur les quantités de DCT consommées localement et les quantités commercialisées par type de circuit, local, national et international. Les données qualitatives sont relatives aux types de relations entre les acteurs de la filière, aux informations qui y circulent et à l'organisation institutionnelle de la filière.

Nous avons donc procédé, durant la période allant de janvier à juin 2019, à des enquêtes, des entretiens et des focus 

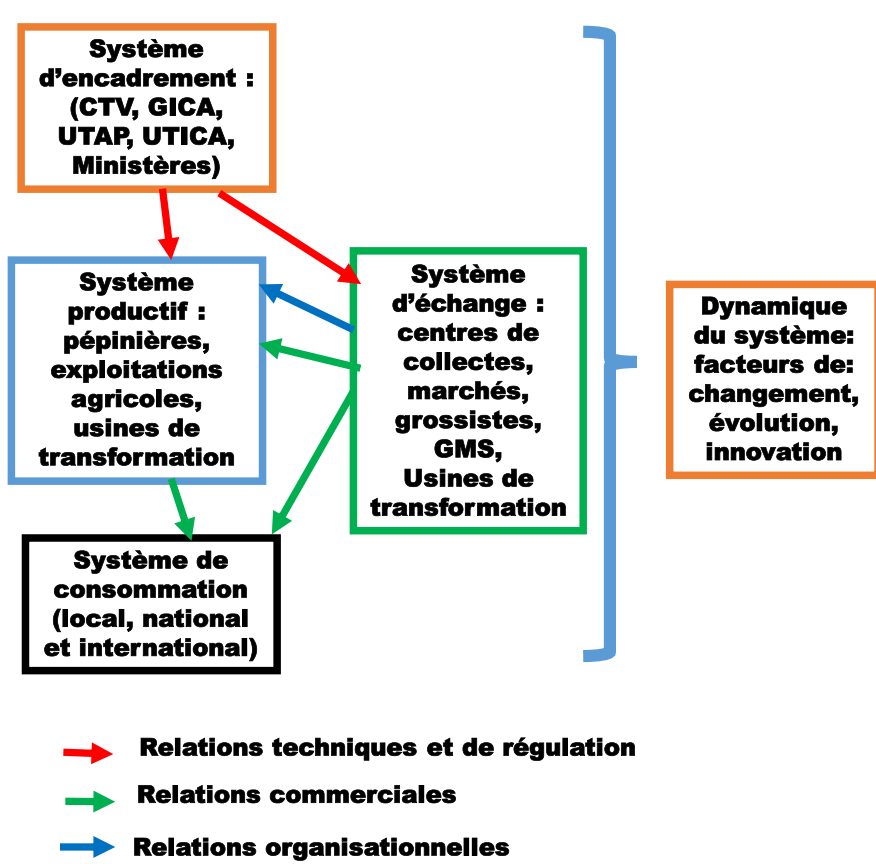

Fig. 4. Systèmes et relations au sein de la filière tomate de transformation dans la plaine de Haouaria en 2019 (source: élaboration des auteurs à partir des résultats des enquêtes).

Fig. 4. System and relations within the tomato supply chain in the Haouaria plain in 2019 (source: development by the authors from survey data).

groupes avec différents acteurs du territoire de Haouaria. Une vingtaine d'agriculteurs ont ainsi été interviewés, pour recueillir des informations qualitatives sur les relations qu'ils entretiennent avec l'amont et l'aval de la filière. Les données quantitatives sur la production de tomates dans le territoire sont celles de la Cellule territoriale de vulgarisation (CTV) de Haouaria. Nous avons aussi interviewé les responsables des quatre usines du territoire sur les quantités de tomates transformées, les volumes et les origines de leurs approvisionnements, et les circuits de distribution de leurs productions de DCT, ainsi que sur leurs relations avec les autres acteurs de la filière. Nous avons réalisé une enquête auprès de 100 consommateurs de la région pour calculer leur niveau de consommation du DCT et pour déterminer l'origine du produit consommé. L'enquête auprès des détaillants a touché 40 personnes présentes sur les principaux marchés de détail de la région pour connaître l'origine de leurs achats. Nous avons également interviewé les responsables de deux associations locales pour discuter de l'impact social et environnemental de la filière. Les entretiens réalisés avec les responsables du Commissariat régional de développement agricole (CRDA) de Nabeul, de la CTV de Haouaria et du Groupement interprofessionnel des conserves alimentaires (GICA), dont le siège est à Tunis, visaient la collecte d'informations quantitatives locales, régionales et nationales et le recueil d'informations qualitatives sur le tissu institutionnel et les modes de fonctionnement de la filière. Enfin, deux focus groupes organisés avec la CTV et constitués de plusieurs acteurs (industriels, administrateurs, consommateurs et agriculteurs) ont été l'occasion de discuter des problématiques locales et de l'avenir de la filière.

\subsection{Types d'analyses}

Plusieurs types d'analyses ont été utilisés en fonction de la qualité et de la précision des données collectées et des objectifs poursuivis. Les enquêtes et entretiens nous ont permis l'identification des réseaux d'acteurs, la construction des maillons et du schéma de la filière ainsi que l'estimation des flux physiques des intrants et des produits au sein de la filière. Les deux focus groupes et les entretiens nous ont permis d'identifier les éléments nécessaires à l'analyse fonctionnelle de la filière. Les données micro-économiques publiées ainsi que les enquêtes et les entretiens réalisés nous ont permis de caractériser les circuits de distribution de la tomate et du DCT et l'étude géographique des flux physiques des deux produits à l'intérieur et en dehors du territoire. L'analyse économique a été basée sur la comparaison de l'évolution des prix de la tomate et du DCT. Enfin, en synthèse de l'ensemble des analyses précédentes, nous avons estimé l'importance quantitative des variables production, distribution et consommation du DCT de cette filière à l'intérieur de son territoire, en comparaison avec le reste du pays.

\section{Résultats}

La filière de la tomate de transformation possède une structure polymorphe avec trois modes de transformation: domestique, artisanal et industriel. Nous appellerons désormais «forme industrielle», la filière de transformation industrielle de la tomate.

En 2018, la forme industrielle dominante a absorbé $90 \%$ des quantités de tomates de saison destinées à la transformation. Seule la forme industrielle produit du DCT. Cette forme contribue de façon importante à l'approvisionnement des marchés aux niveaux local, national et étranger. L'autoconsommation en tomate a été estimée à $5 \%$ de la production de la région en 2018 (nos enquêtes consommateurs et agriculteurs et entretiens CTV)

Notre enquête consommateurs réalisée dans la région a montré que la consommation totale en équivalent tomates fraîches a été de $146 \mathrm{~kg}$ par personne en moyenne en 2018 et que $75 \%$ de cette quantité a été consommée sous forme de DCT, soit l'équivalent de $16,6 \mathrm{~kg}$ par personne. Ce produit, très important dans la cuisine tunisienne, coûte moins cher que les tomates fraîches. Ainsi, le budget destiné à la consommation de DCT a représenté environ $40 \%$ du budget de la consommation de tomates dans la région (nos enquêtes consommateurs). Le DCT, produit exclusif de la forme industrielle, est ainsi le plus important en termes de consommation. Ceci nous amène à focaliser notre travail sur cette forme dominante de la filière en termes de consommation, production, transformation et échanges.

Pour mieux comprendre les mécanismes de régulation de cette filière et leurs impacts locaux, nous présentons d'abord la structure de la forme industrielle, ensuite son fonctionnement et les mécanismes de sa régulation.

\subsection{Structure de la forme industrielle}

La filière tomate de transformation de ce territoire est composée de différents systèmes fonctionnels de production, 


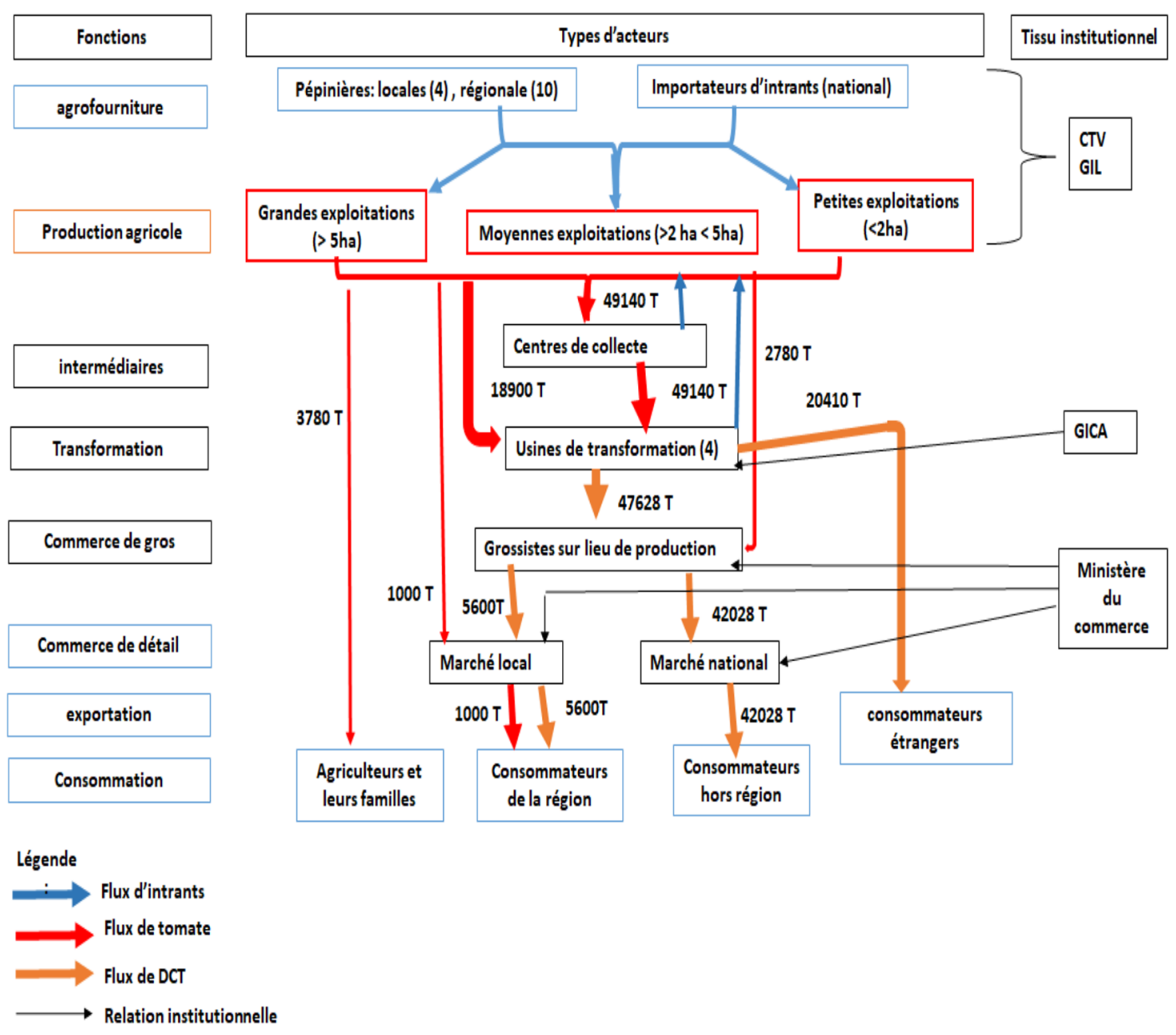

Fig. 5. Schéma de la filière tomate de transformation à Haouaria (données de 2018) (source : élaboration des auteurs à partir des données des enquêtes).

Fig. 5. Diagram of the tomato supply chain in the Haouaria plain (2018 data) (source: development by the authors from survey data).

d'échange, d'encadrement et de consommation. Ces systèmes ont différents types de relations dynamiques entre eux et avec l'extérieur (Fig. 4).

Le système de production interne (Fig. 5) comprend des pépinières installées par des promoteurs privés (14 sites de production), des exploitations agricoles (environ un millier) et des usines de transformation (4 usines). La production prévisionnelle des pépinières de la région était de 422 millions de plants de tomates en 2019 (nos entretiens CTV). Ce potentiel permettait la plantation de plus de 21000 hectares. Ces pépinières approvisionnent tout le Cap Bon et la majorité des régions productrices de tomate de Tunisie. Cette production est majoritairement destinée aux exploitations des autres territoires.

Le système agricole est formé majoritairement par de petites exploitations agricoles dont la superficie moyenne est inférieure à 1 ha. Les exploitations ne sont pas regroupées dans des structures professionnelles. Les exploitants ont de faibles moyens financiers. Pour la majorité, le financement de la campagne de tomate se fait sous forme d'avances par les centres de collecte et les industriels. Les exploitants s'engagent par conséquent à leur vendre leur production. Dans la plaine, le recours à de la main-d'œuvre extra-régionale est intense durant les périodes de récolte de la tomate et se fait dans des conditions de travail très rudimentaires. Enfin, quatre grandes usines de transformation existent dans le territoire depuis les années 1970. En 2018, leur capacité de transformation de la tomate représentait la moitié de celle du Cap Bon et le quart de celle de la Tunisie. Elles ont également assuré $40 \%$ des exportations de DCT de la Tunisie pour la même année. Ces usines sont certifiées et répondent aux normes internationales de qualité (GICA, 2020). Pour leurs approvisionnements, elles s'adressent de plus en plus à des exploitations situées en dehors de la plaine.

Le système d'échange (Fig. 5) est formé par l'ensemble des acteurs, depuis la distribution des intrants jusqu'à la distribution des dérivés de la tomate. Dans la plaine de Haouaria, on identifie des centres de collectes (une quarantaine), des grossistes (une centaine) et des détaillants (1230 points de ventes) (CGDR, 2019). Les centres de collecte interviennent comme intermédiaires entre les industriels et les agriculteurs pour la culture et la collecte de la tomate. Ils ont assuré $65 \%$ des approvisionnements en tomates des usines de la région en 2018. Ils s'occupent également de la diffusion du 


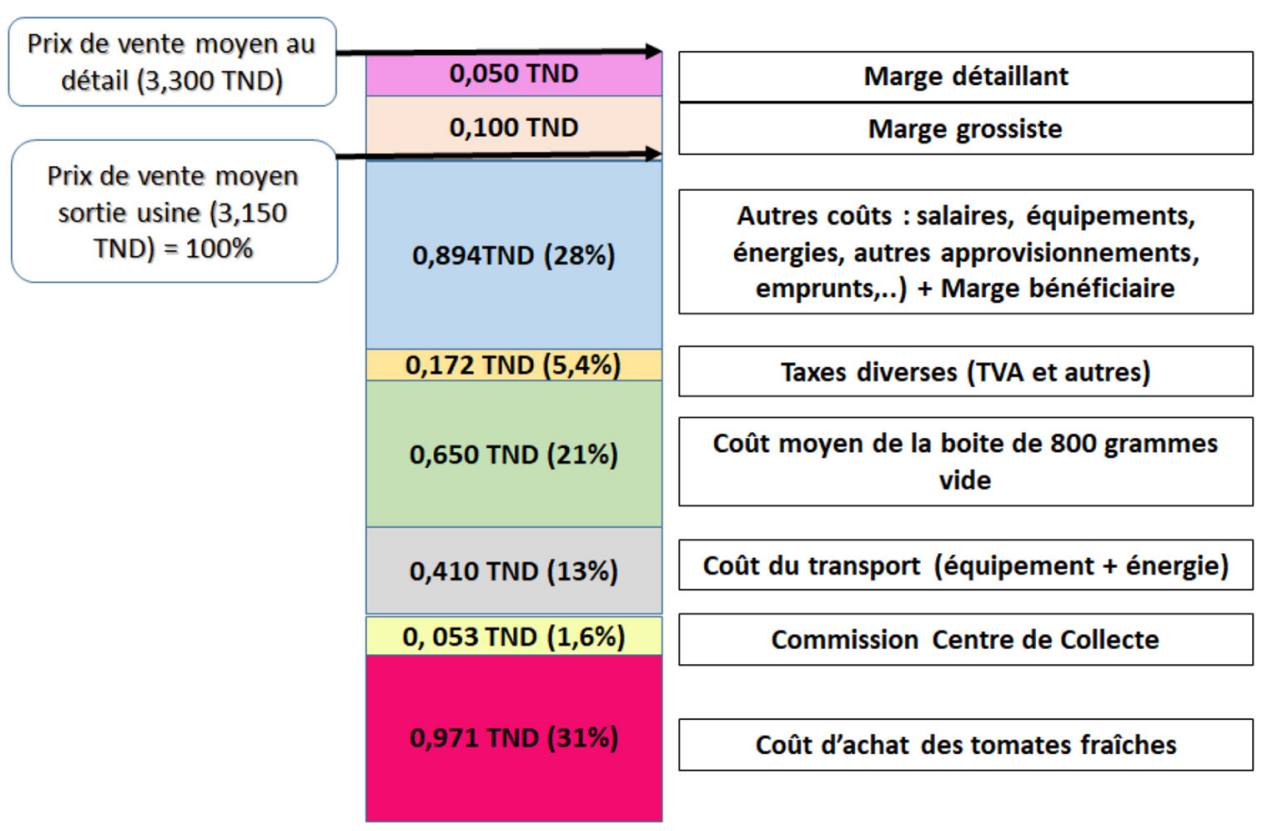

Fig. 6. Structure des coûts d'une boîte de $800 \mathrm{~g}$ de Double Concentré de Tomate «DCT» (estimation des auteurs, 2020).

Fig. 6. Cost structure of an $800 \mathrm{~g}$ box of double tomato concentrate (authors estimation, 2020).

paquet technique auprès des agriculteurs. Avant la Révolution, ces centres étaient soumis à des cahiers des charges et au contrôle qualité. Leur nombre a presque doublé depuis le début des années 2010 (entretien GICA). Leur situation est actuellement différente avec un travail beaucoup plus informel (entretiens GICA et industriels). Leurs responsables développent des liens personnels avec les usines de transformation (entretiens industriels). Ces centres s'approvisionnent aussi bien auprès des agriculteurs de la plaine qu'auprès des agriculteurs d'autres régions. Par ailleurs, plusieurs grossistes de la région et en dehors de celle-ci interviennent dans la distribution de la tomate et du DCT. Les quatre usines de transformations du territoire cumulent parfois la fonction de vente en gros et de livraison des détaillants.

Le système d'encadrement (Fig. 5) est formé par la CTV qui intervient auprès des petits agriculteurs pour leur apporter un conseil technique. Elle offre aussi un service de contrôle qualité auprès des pépinières et des unités de vente des intrants agricoles. Elle a un rôle de collecte et de diffusion de l'information entre les différents agents de la filière et pour l'administration de tutelle. Une commission nationale de préparation et de suivi de la filière est formée par des représentants du GICA, de l'Union tunisienne de l'agriculture et de la pêche (UTAP) et de l'Union tunisienne des industries, du commerce et de l'artisanat (UTICA). Elle décide, au début de chaque campagne de culture de la tomate, des besoins en superficies à cultiver en se basant sur les besoins du marché tunisien en DCT, les prévisions d'exportation de DCT et le niveau des stocks de ce produit. Cette commission fixe également le prix de vente de la tomate aux usines de transformation. Le GICA offre aussi une assistance technique et un service d'analyse de la qualité et d'agrémentation aux usines de transformation. Il participe aux salons internationaux pour promouvoir les marques tunisiennes. Ce système est donc principalement composé de professionnels externes au territoire et dont les préoccupations sont essentiellement méso et macro-économiques.

Les acteurs de ces différents systèmes entretiennent des relations multilatérales et font des choix qui influent sur le fonctionnement et la régulation de la filière. Les liens de la filière avec sa région ne se limitent pas aux facteurs pédoclimatiques mais s'étendent à des variables sociales, économiques, environnementales et patrimoniales (Barjolle et al., 1998), comme nous allons le voir dans ce qui suit.

\subsection{Fonctionnement et mécanismes de régulation}

Les acteurs des sous-systèmes fonctionnels entretiennent différents types de relations, entre eux et avec l'extérieur, qui caractérisent le fonctionnement et la régulation de la filière.

On distingue d'abord les relations techniques qui sont formelles, par contrats de culture, ou informelles et qui lient les agriculteurs aux centres de collecte ou aux usines de transformation. Dans le cadre de ces relations, l'agriculteur reçoit les intrants et un paquet technique à exécuter. Il s'engage à vendre sa récolte à celui qui finance son approvisionnement en intrants.

Le financement de la culture de tomate par des tiers est un mécanisme de régulation important dans cette filière. L'exploitant agricole, pour diverses raisons, n'a plus les moyens d'assurer lui-même le financement de la culture de la tomate de transformation, estimé entre 7 et 8000 TND par hectare en 2019 (nos enquêtes agriculteurs et entretiens CTV et industriels) $(1$ euro $=3,27$ TND en juin 2019). Le système de production antérieur de la plaine était basé sur le maraîchage et l'élevage, qui a été abandonné à cause du renchérissement du coût de l'alimentation animale (nos enquêtes agriculteurs). L'élevage était cependant une source importante de financement de la culture de tomate. 
Dans le modèle de production actuel, l'exploitant apporte uniquement la terre et sa force de travail. Les intrants utilisés, les choix techniques et la production ne lui appartiennent plus. La rémunération de l'agriculteur reflète la faible intégration dans la filière puisque la valeur de la tomate achetée aux agriculteurs ne dépasse pas $31 \%$ du prix de vente du DCT (Fig. 6).

Les relations organisationnelles expriment l'intégration, la coopération et/ou la concurrence entre les différents maillons de la filière. L'intégration verticale des industries de transformation est forte. Certaines cumulent, avec la fonction de transformation, celle d'approvisionnement et de distribution aux stades de gros et de détail. Les centres de collecte sont également fortement intégrés à l'amont dans l'activité agricole. Les relations de coopération existent entre les pépinières, les centres de collecte et les industries de transformation pour l'approvisionnement des agriculteurs en plants. La concurrence est par contre très importante au niveau du maillon de transformation, surtout pour l'approvisionnement en tomates. Les quatre usines du territoire cherchent à minimiser les coûts de leurs achats et préfèrent acheter la tomate du territoire, qui de plus est réputée pour sa meilleure qualité grâce à un Brix (pourcentage de sucre soluble) élevé.

Enfin, les relations d'échange de la filière sont principalement marchandes et formalisées au niveau des pépinières et des usines de transformation. Elles sont principalement informelles pour les exploitations agricoles et les centres de collectes.

La tomate de transformation est vendue soit dans des circuits courts, aux usines de transformation à travers les centres de collecte ( $65 \%$ des quantités), soit dans des circuits directs exploitation-usine ( $25 \%$ des quantités) (Fig. 5). Les moyens de commercialisation des agriculteurs de la plaine de Haouaria étant faibles, ils vendent la majorité de leur production $(90 \%)$ aux centres de collecte et aux usines de transformation (Fig. 5), qui disposent d'importants moyens logistiques.

Les prix de vente de la tomate fraîche destinée à la transformation sont fixés, au début de chaque campagne, par une commission nationale regroupant l'UTAP, l'UTICA, les ministères de l'agriculture et de l'industrie et le GICA. Le niveau des prix demeure faible. En 2020, les agriculteurs ont vendu le kilo de tomate de transformation à $0,184 \mathrm{TND}$, contre $1 \mathrm{TND}$ en moyenne sur les marchés de détail pour la consommation en frais.

D'un autre côté, les circuits de distribution du DCT en Tunisie sont longs. En 2018, $70 \%$ des quantités produites dans la plaine de Haouaria sont passées par des grossistes et des centrales d'achat. Seulement $8 \%$ de la production locale de DCT a été vendue sur place, $62 \%$ sur le marché national et $30 \%$ a été destinée à l'exportation. Par ailleurs, $87 \%$ des quantités de DCT consommées dans la région proviennent des marques locales (Fig. 5).

La régulation de la forme industrielle de cette filière a été pilotée par différents acteurs et par des décisions politiques ces 20 dernières années. Cette régulation s'est orientée dans des sens et des rythmes différents en fonction du maillon considéré. Ainsi, les observations suivantes ont pu être faites.

$\mathrm{La}$ régulation de la production de la tomate de transformation dans la plaine de Haouaria a été opérée grâce à l'intervention de l'État, par des primes en 2010-2013 pour inciter les agriculteurs à réduire les superficies destinées à cette culture. Cette mesure a été décidée à cause de la baisse des disponibilités en eau dans la plaine. La concurrence locale sur l'eau pour toutes les cultures maraîchères et l'entrée en production d'autres régions pour la tomate de transformation ont rendu cette décision possible. Cela c'est alors traduit, dans la plaine, par la stagnation des superficies cultivées en tomates depuis 2014. Néanmoins, une autre baisse des superficies est prévisible suite aux récentes orientations des politiques agricoles par l'adoption de subventions pour la plantation d'oliviers dans le territoire. De même, un processus de reconversion serait en cours grâce à l'investissement dans le développement des pépinières, stimulé par une demande en plants en croissance dans tout le pays. Par ailleurs, les terres agricoles de la plaine subissent de plus en plus la forte pression de l'urbanisation favorisée par l'extension du réseau routier et les constructions de logements en bord de mer et à l'intérieur de la plaine (entretiens CTV).

Toutefois, la tomate produite dans le territoire de Haouaria demeure recherchée pour sa bonne qualité organoleptique, comparée avec celle des tomates produites en dehors du Cap Bon (enquêtes agriculteurs et entretiens industriels et CTV). En effet, la culture de la tomate bénéficie des conditions édaphiques et climatiques du Cap Bon et fournit un fruit dont le Brix est le plus élevé de Tunisie avec une maturité tardive recherchée par les industriels. Cette qualité permet le maintien de l'intérêt pour cette culture dans la plaine tant que les disponibilités en eau le permettent.

La sécurisation de l'approvisionnement des usines de transformation se fait par des contrats et des avances aux cultures par l'intermédiaire des centres de collecte, ou de façon directe par les industriels. En effet, les agriculteurs de la plaine n'ont plus les moyens de financer leur culture de tomate, les coûts de production étant trop élevés et avoisinant 7000 à 8000 dinars par hectare en 2019 (enquêtes agriculteurs). Ces coûts de production ont progressé à des rythmes très importants. En effet, tous les intrants importés (semences, fertilisants, produits de traitements, énergie, emballage) ont subi la dévaluation du dinar $(13,5 \%$ en moyenne annuelle entre 2010 et 2018). Le coût de la main-d'œuvre a également augmenté à un taux annuel moyen de $18 \%$ pour la même période. En contrepartie, les prix de la tomate n'ont progressé que de 4,2\% entre 2010 et 2018 . En parallèle, durant la même période, les agriculteurs ont vu leur trésorerie se détériorer à cause de l'abandon de l'élevage, devenu non rentable suite à la forte augmentation des coûts des aliments importés (enquêtes agriculteurs et entretiens CTV). Or l'élevage a longtemps joué le rôle de régulateur de la trésorerie pour le financement des activités maraîchères (enquêtes agriculteurs). L'abandon de l'élevage a entraîné une plus grande dépendance des agriculteurs aux financements externes.

Par ailleurs, dans la plaine de Haouaria, la baisse des superficies cultivées en tomates (Fig. 2) a entraîné une forte concurrence pour l'approvisionnement entre les usines de transformation, rendant nécessaire la fidélisation des agriculteurs.

La sécurisation de l'approvisionnement a orienté les industriels vers les ressources externes à la plaine par le recours à d'autres régions productrices de Tunisie. Les systèmes des avances et des contrats de culture, ainsi que le recours aux centres de collecte, sont les mêmes pour les agriculteurs en 
dehors de la plaine. Les industriels cherchent ainsi à minimiser les risques de leurs approvisionnements par le financement des agriculteurs et la création d'une relation de dépendance, la concurrence par les prix n'étant pas possible puisqu'ils sont fixés au début de chaque campagne de production de la tomate.

Par conséquent, durant la dernière décennie, le système d'approvisionnement des usines de la plaine s'est fortement ouvert aux autres gouvernorats, dans un processus de déterritorialisation. Ainsi, en 2018, les usines de transformation de Haouaria se sont approvisionnées à hauteur de $70 \%$ à $90 \%$ en dehors de leur territoire (entretiens industriels). Cette ouverture a été également accompagnée par une diversification des marchés pour les pépinières de la région.

Cette industrie de proximité offre, en plus d'un produit local fortement consommé, des emplois et des revenus pour la population du territoire.

Un effort d'innovation à travers l'importation de nouvelles variétés et de nouveaux produits dérivés de la tomate (purée, concassée, en sauces...) émerge depuis quelques années. L'innovation concerne aussi des emballages plus faciles d'utilisation et l'acquisition de techniques de transformation plus modernes.

Les industriels ont aussi investi dans l'amélioration de leur empreinte écologique par l'installation de stations d'épuration des eaux usées, l'utilisation du gazole en tant qu'énergie plus propre en remplacement du fuel lourd et le recyclage des déchets solides dans l'alimentation animale (entretiens industriels).

\section{Conclusion et discussion}

La forme industrielle de la filière tomate de transformation dans la plaine de Haouaria s'est développée grâce à des investissements d'innovation dans les produits et les procédés au niveau des pépinières et des industries de transformation. Néanmoins, cette forme s'est éloignée de la filière de proximité qui a été développée à partir des années 1970. En effet, son développement s'est accompagné d'une déterritorialisation progressive de son approvisionnement, à cause des pressions sur le secteur agricole liées aux ressources en eau. Son extraversion a également été importante pour l'écoulement de sa production. Cette filière s'est donc développée, depuis le début des années 2010, grâce à certaines ressources externes à son territoire, tout en mobilisant le capital humain, le savoirfaire et les ressources financières internes. Cette dynamique devrait se maintenir puisque les récentes mesures de politique agricole dans la plaine orientent l'activité agricole vers l'oléiculture, d'où une plus grande concurrence pour les ressources naturelles locales. Ainsi, une reconversion agricole, même partielle, de la région, affaiblirait encore plus l'ancrage territorial de la filière tomate de transformation par une baisse de la disponibilité de la tomate locale.

Par ailleurs, dans ce territoire, les asymétries de pouvoir des acteurs sont importantes. En effet, le pouvoir financier des agriculteurs est très faible, ce qui les rend vulnérables et soumis aux décisions des industriels dont le pouvoir financier est le plus fort. Cette asymétrie éloigne les acteurs économiques des valeurs liées à une filière territoriale et au développement local inclusif, qui devraient être le socle d'un engagement territorial pour une durabilité de la filière.
Les mécanismes de régulation mis en œuvre au niveau micro-économique concernent la sécurisation de l'approvisionnement et la réduction de l'impact sur l'environnement pour les usines de transformation, et la diversification des marchés pour les pépinières et les usines de transformation. Dans ce processus de régulation, nous avons pu relever que la connexion entre les opérateurs de la filière et ceux du territoire est de plus en plus faible, accentuant la déterritorialisation de la filière.

La régulation de cette filière est également, en grande partie, administrée aux niveaux méso et macro-économique par la profession et l'État, qui interviennent dans la fixation du prix des tomates fraîches destinées à la transformation, du prix de l'énergie, des marges de détail et de gros. Ceci témoigne d'une autre forme de déconnexion entre les centres de décision et ceux de production.

Par ailleurs, et concernant la durabilité environnementale de la filière, la forme industrielle présente une empreinte écologique assez forte, mais en amélioration, notamment pour les activités de transformation. Ainsi, le recyclage des déchets solides des usines vers l'alimentation animale, le traitement des eaux usées et la reconversion au gazole au lieu du fioul lourd auraient amélioré l'empreinte écologique des industries de transformation dans la région (entretiens industriels). Néanmoins, le recours grandissant à l'approvisionnement en dehors de la région entraînera l'augmentation de l'empreinte carbone, par le transport, et de l'empreinte sociale, par une baisse de l'emploi agricole local. Ceci mérite d'être analysé. De plus, concernant l'activité agricole, l'empreinte écologique est en hausse suite à la surexploitation des ressources hydriques, à l'augmentation de la salinité de l'eau et à la dégradation conséquente des terres agricoles. L'orientation de la politique locale vers l'oléiculture serait-elle la meilleure solution pour cette plaine? Des études d'impacts socio-économiques et écologiques seraient nécessaires pour en discuter.

Enfin, dans la plaine de Haouaria, les acteurs revendiquent dans leurs discours une qualité territoriale de la tomate (enquêtes agriculteurs et entretiens CTV et industriels), mais n'ont entamé aucune action de différenciation de leur produit. Aucun acteur ne cherche à s'identifier de façon formelle à la région. Le mélange de la tomate locale avec les produits des autres régions, au stade de la transformation, lui fait perdre son appartenance. Cependant, dans la plaine, l'alternative de la transformation artisanale en tomates séchées prend de l'importance. Ce produit est commercialisé dans des circuits courts qui valorisent mieux la tomate locale et qui méritent d'être étudiés. L'émergence de cette nouvelle filière pourrait-elle à l'avenir pérenniser la culture locale de tomate et valoriser durablement les ressources territoriales? Des études sociales, économiques et environnementales seront nécessaires pour le dire.

Financement. Cette recherche a été soutenue par le projet ARIMNET2 «DIVERCROP» «Land system dynamics in the Mediterranean basin across scales as relevant indicator for species diversity and local food systems ».

\section{Références}

Barjolle D, Boisseaux S, Dufour M. 1998. Le lien au terroir. Lausanne (Suisse): Institut d'économie rurale, ETHZ, Antenne romande, 33 p. 
Bencharif A, Rastoin JL. 2007. Concepts et méthodes de l'analyse de filières agroalimentaires : application de la chaîne globale de valeur au cas des blés en Algérie. Montpellier (France): Working paper MOISA $n^{\circ} 7 / 2007$, UMR MOISA: CIHEAM-IAMM, CIRAD, INRAE, Institut Agro, Montpellier SupAgro, IRD, 24 p.

CGDR (Commissariat Général au Développement Régional). 2019. Gouvernorat de Nabeul en chiffres. Tunis (Tunisie), 200 p.

Champion C. 2014. Liens entre organisation des filières et transferts nutritionnels : le cas du double concentré de tomate en Tunisie. Montpellier (France) : Mastère UMR MoISA : CIHEAM-IAMM, CIRAD, INRAE, Institut Agro, Montpellier SupAgro, IRD, 144 p.

Cheriet F. 2015. Filières agroalimentaires et chaînes globales de valeur : concepts, méthodologies et perspectives de développement. Montpellier, France : Working Papers MOISA 209883, Institut National de la Recherche Agronomique (INRA), UMP 1110 MOISA, 10 p.

CTV (Cellule Territoriale de Vulgarisation). 2019. El Haouaria: données 2019. Tunis (Tunisie): Ministère de l'Agriculture, $20 \mathrm{p}$.

Ferchichi I, Mekki I, Elloumi M, Arfa L, Lardon S. 2020. Actors, scales and spaces dynamics linked to groundwater resources use for agriculture production in Haouaria plain, Tunisia. A territory game approach. Land 9(74): 15. https://doi.org/10.3390/land9030074.

GICA (Groupement des industries de conserves alimentaires). [ 2020/ 12/20]. http://www.gica.tn/fr.
Griffon M. 2002. Agriculture, écologie et équité, une trajectoire à poursuivre. In: Dufumier M, ed. Un agronome dans son siècle: actualité de René Dumont. Paris (France) : Karthala, pp. 187-194.

Madelrieux S, Buclet N, Lescoat P, Moraine M. 2017. Caractériser les formes d'interaction entre filières agricoles et territoires: quelles méthodes? Cahiers Agricultures 26(2): 24002. https://doi.org/ $10.1051 /$ cagri/2017014.

Manouvrier E. 2008. Une filière en recomposition et ses incidences territoriales : l'endive dans la France du Nord. Revue d'Économie Régionale \& Urbaine 2: 211-224. https://doi.org/10.3917/ reru.082.0211.

Rastoin JL, Ghersi G. 2010. Le système alimentaire mondial. Concepts et méthodes, analyses et dynamiques, l'analyse de filières agroalimentaires. Versailles (France) : Éditions Quae, pp. 121-192. https://doi.org/0.3917/quae.rasto.2010.01.

Temple L, Lançon F, Palpacuer F, Paché G. 2011. Actualisation du concept de filière dans l'agriculture et l'agroalimentaire. Économies et Sociétés, Série AG Systèmes agroalimentaires. Presses de l'ISMEA, 33, pp. 1785-1797.

Tozanli S, El Hadad-Gauthier F. 2007. Gouvernance de la chaîne globale de valeur et coordination des acteurs locaux : la filière d'exportation des tomates fraîches au Maroc et en Turquie. Cahiers Agricultures 16(4): 278-286. https://doi.org/10.1684/agr.2007.0110.

Citation de l'article : Arfa L, Elloumi M. 2021. La filière tomate de transformation à Haouaria en Tunisie: prédominance de la forme industrielle déterritorialisée. Cah. Agric. 30:31. 